\title{
The Adopted Morphological Types of 247 Rich PF Galaxy Clusters
}

\author{
Elena Panko ${ }^{1}$, Katarzyna Bajan ${ }^{2}$, Piotr Flin ${ }^{3}$ and Alla Gotsulyak ${ }^{4}$ \\ ${ }^{1}$ Kalinenkov Astronomical Observatory, Nikolaev National University, Nikolaev, Ukraine \\ email: panko.elena@gmail.com \\ ${ }^{2}$ Institute of Physics, Pedagogical University, Cracow, Poland, \\ ${ }^{3}$ Institute of Physics, Jan Kochanowski University, Kielce, Poland \\ ${ }^{4}$ Astronomical Department, Odessa National University, Odessa, Ukraine
}

\begin{abstract}
Morphological types were determined for 247 rich galaxy clusters from the PF Catalogue of Galaxy Clusters and Groups. The adopted types are based on classical morphological schemes and consider concentration to the cluster center, the signs of preferential direction or plane in the cluster, and the positions of the brightest galaxies. It is shown that both concentration and preferential plane are significant and independent morphological criteria.
\end{abstract}

Keywords. Galaxies: clusters: morphological types.

\section{Introduction}

The classification of galaxy clusters at optical wavelengths is carried out using several different parameters: cluster richness (number of galaxies within a specific limiting magnitude), degree of central concentration, the presence of bright galaxies in the center of the cluster, etc. The prevalent Bautz-Morgan (BM) (Bautz \& Morgan, 1970) and Rood-Sastry (Rood \& Sastry, 1971) classification schemes are in agreement and complement each other. López-Cruz et al. (1997) introduced the definition of a cD cluster, the complement of which is called a non-cD cluster.

\section{Observational Data}

A Catalogue of Galaxy Clusters and Groups (Panko \& Flin, 2006, hereafter PF) was constructed from the Münster Red Sky Survey Galaxy Catalogue (Ungrue, Seitter \& Duerbeck, 2003, hereafter MRSS) mainly for statistical analysis of properties for largescale structures. Unfortunately, so far we have only been able to study the cluster parameters from the morphology for $1056 \mathrm{PF}$ clusters that are coincident with those in the ACO catalogue (Abell, Corvin \& Olovin, 1989). Similarly, only 247 PF clusters with richness $N \geqslant 100$ have assumed BM morphological types according to the ACO catalogue. Those morphological types permit us to find alignments of the brightest galaxy relative to the parent clusters for BM type I (Panko, Juszczyk \& Flin, 2009). The Binggeli effect (Binggeli, 1982) is strongest for BM type I clusters, as well presents for BM III clusters (Flin et al., 2011). Moreover, Godłowski et al. (2010), using data for 97 PF galaxy clusters, found a weak dependence of galaxy velocity dispersion with BM type for the parent cluster. Other morphology schemes for PF clusters were not used. Presently we adopted the prevalent morphological systems for the MRSS observational data and determined our morphological types for PF galaxy clusters using a $2 D$ distribution of galaxies in rectangular coordinates relative to the cluster center for each. 

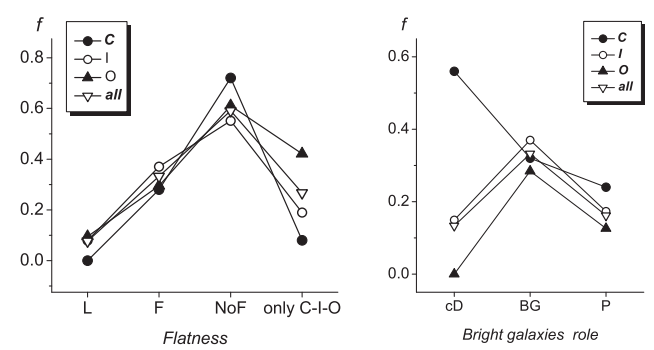

Figure 1. Variation of frequencies for flatness signs (left) and BCM role in C-I-O and all clusters.

\section{The adopted morphological scheme}

For input data we established adopted morphological types based on 3 parameters: concentration, signs of flatness, and bright cluster members (BCM) positions. The adopted types correspond to concentration ( $\mathrm{C}$ - compact, I - intermediate, and $\mathrm{O}$ - open), flatness ( $\mathrm{L}$ - line, $\mathrm{F}$ - flat, and no symbol if no indication of flatness is present), and the role of bright galaxies (cD or BG if the BCM role is significant). Other peculiarities are noted as P. The details of the approach are described and justified elsewhere Panko (2013). The designations can be combined, for example CFcD or ILP.

For 247 rich PF clusters with BM types from the ACO comparison, we determined the adopted morphological types and analyzed the frequencies of each. The sign of the flatness type is independent of concentration class: those for $\mathrm{L}$ and $\mathrm{F}$ types are similar in C-I-O groups, as shown in Fig. 1, left panel. In contrast, the role of BCMs is strongly connected with cluster concentration: the number of $\mathrm{cD}$ clusters is greatest in C-type (Fig. 1, right panel). Note, CcD type corresponds to BM I type. For L and F clusters we found a correlation between position angle for the major axes of the best-fit ellipse and the direction of the preferred plane.

\section{Conclusions}

From $2 D$ maps of 247 rich $\mathrm{PF}$ galaxy clusters we determined their adopted morphological types. It is shown that concentration and flatness are independent morphological criteria. The direction of the major axis of the best-fitting ellipse for a cluster (calculated in the PF catalogue) is close to the direction determined by the $\mathrm{L}$ or $\mathrm{F}$ regions; the difference between the two directions increases for O-type galaxy clusters.

\section{References}

Abell, G. O., Corwin, H. G., \& Olowin, R. P. 1989, ApJS, 70, 1

Bautz, P. \& Morgan, W. W. 1970, ApJ, 162, L149

Binggeli, B. 1970, $A \& \exists$ A, 162, L149

Flin, P., Biernacka, M., Godlowski, W., et al. 2011, Balt. Astr., 20, 251

Godłowski, W., Piwowarska, P., Panko, E., et al. 2011, ApJ, 723, 985

López-Cruz, O., Yee, H. K. C., Brown, J. P., et al. 1997, ApJ, 475, L97

Panko, E. 2013, Odessa Astr. Publ., 26, 90

Panko, E. \& Flin, P. 2006, Jornal of Astronomical Data, 12, 1

Panko, E., Juszczyk, T., \& Flin, P. 2009, AJ, 138, 1709

Rood, H. J. \& Sastry, G. N. 1971, PASP, 83, 313

Ungrue, R., Seitter, W. C., \& Duerbeck, H. W. 2003, Jornal of Astronomical Data, 9, 1 